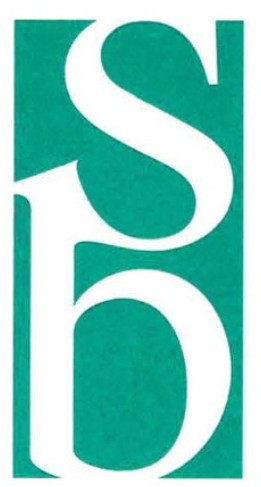

\title{
Battle for BMRB
}

Washington $D C$ - The international NMR community is in a furor following the recent unofficial decision of the National Institutes of Health's National Library of Medicine (NLM) not to fund the proposal for the continued maintenance and expansion of the sole (NMR) spectroscopy database for macromolecular structures.

The Biological Magnetic Resonance Data Bank (BMRB) initiative at the University of Wisconsin at Madison was started in an attempt to meet the data storage needs of the growing NMR research community. The originators of the project, John Markley and Eldon Ulrich, were given a research grant in 1988 to develop the database for research purposes by the NLM.

Ulrich and Markley say that, although BMRB has submitted three proposals to NLM since 1993 for the increased funding needed to make the database fully operational, none of them has been approved, and the database has been maintained instead at a constant funding level through administrative 'supplementals' to the original grant.

BMRB has received further support in the form of a subcontract from the Brookhaven Protein Data Bank (PDB) and Department of the Energy to facilitate collaboration between the databanks. The aim, Ulrich says, is to devise common formats for distributing NMR (and X-ray) data, providing direct links between the data in the two databases and providing the PDB with NMR expertise. Professor Joel Sussman, Head of the PDB, confirms that the two data banks have been working in close collaboration to develop methods for processing information derived from both NMR and X-ray crystallographic studies.

The rise of NMR spectroscopy in the last decade as a means of determining the highresolution structures of biological macromolecules has presented new data storage problems, as the data are fundamentally different from those generated by X-ray crystallography. The raw data of the NMR spectra yield so-called 'primary data': chemical shifts, coupling constants, relaxation parameters, and so on. These, in turn, are assigned and used to generate the list of constraints that ultimately defines the three-dimensional structural models of the molecule being studied.

NMR-based coordinate files and constraint lists can be deposited at the PDB (although Sussman notes that there is no standard format for handling the constraint lists at the moment) but there is no facility to store the primary data. These data are not only important as a means of independently validating the structure determination (like X-ray structure factors) but are also a rich source of information on a variety of aspects of protein structure including dynamics, hydrogen bonding and local electric-field gradients.

In the hope of gearing up the database in the face of the increase in the number of structures determined by NMR and in anticipation of the continuing expansion of the use of the technique-the PDB now has coordinates for $\sim 470$ NMR-derived structures with the rate of deposition increasing by $20-30 \%$ a yearMarkley, Ulrich, Miron Livny and Yannis Ioannidis submitted a proposal last November to NLM to: expand the database staff from the present two-and-a-half to five members; develop an interface capable of dealing effectively with complex and varied NMR datasets; and to acquire additional computer hardware.

The proposal received a "good, but not stunning" priority rating, says Markley. Nonetheless, Markley and colleagues believed that they would receive the funding to maintain and develop the database further. Thus it was with some shock and dismay, Ulrich records, that they discovered informally that the databank had not been funded: the (unofficial) reason is simply that the money for the 1995 fiscal year (which begins on 1 October) ran out before the BMRB proposal was reached on the priority list.

Conflicting signals are coming from the 
NLM regarding the basis of the review board's priority score for the BMRB proposal, the critical parameter in funding decisions. On one hand, the score seems to have been based to some degree on the extent of the present use of use the database, measured in 'hits' (people visiting/using the database) per day — a mere dozen or so, some three orders of magnitude removed from the level of use the NLM would expect for such a database, according to an unnamed source. Dr Robert Dahlen, Chief of the Bioinformation Support Branch, Extramural Program at the NLM confirms that the review board was concerned about whether the database was sufficiently "used or useful". On the other hand, Milton Corn, MD, Associate Director, Extramural Program at the NLM, says that there were problems "with the sophistication of the [database] proposal".

Although the current funds ran out on August 1 the NLM has promised to provide the databank with a small amount of money $(\$ 70,000)$ to "limp along", Dahlen says. Certainly the database will not limp for long on $\$ 70,000$; Ulrich predicts about two to three more months, at the very most. As an unnamed leading NMR structural biologist points out, the money is suitable only for "an orderly shutdown" of the database, a point confirmed by Corn.

The NLM's decision has caused considerable consternation in the NMR community. The Internet has been buzzing with news of the possible closure and an informal campaign of protest has sprung up, consisting mainly of letter-writing to Dahlen, who admits that his office has been deluged with letters and faxes protesting at the lack of funding for BMRB.

At one level Markley and Ulrich seem to be victims of their forward thinking. NMR as a tool for determining macromolecular structure is still in its infancy, and a critical mass of users is only beginning to develop. Ad Bax, one of the leading NMR structural biologists at the $\mathrm{NIH}$, points out that the "role of the BMRB may well change in the next few years" and anticipates that once the database has grown approximately fivefold "it will contain the minimum critical mass of data needed to do rigorous statistical checking" of structure parameters. Once BMRB has grown sufficiently and includes de- tails of relaxation parameters, Bax believes "it may well contain sufficient information to find the key which translates structure and dynamics into chemical shift".

The database is also seen as being important from a technical point of view. Bax recounts that the discovery of the correlation between alpha-carbon chemical shifts and phi/psi angles was critically dependent on the existence of NMR databases, both BMRB and a local database at the NIH. And Gerhard Wagner, at Harvard Medical School, points out that a centralized database is essential for the field "to cross the threshold from manual and visual data inspection to automated data analysis". Herein lies the 'Catch-22'; automation would, of course, fuel further growth and development of the databank and undoubtedly increase the both the hits-per-day rating and database sophistication - of which the present proposal seems to have fallen foul.

Efforts are being made behind the scenes at the NIH to secure funds to maintain the database on a long-term basis in response to the outcry from the NMR community, unnamed sources say. Certainly Markley and colleagues are considering alternate sources of funding within the NIH as well as putting forward another proposal at the beginning of the 1996 fiscal year when, presumably, they will not face the problem of funds drying up quite so quickly.

The reprocussions of the failure to fund the BMRB are, Sussman warns, "disturbing" and "will significantly hinder the ability of the PDB and the future Macromolecular Structure Database to [either] archive accurately NMR data [or] provide adequate service to the NMR structure community".

Indeed, the whole issue of how databases should be funded is being looked at very closely, says Corn. Those not supported by the Government may well have to be privatized, he believes. Nonetheless, Ulrich is strongly of the belief that "the database must be publicly, and freely accessible"; he does not want it "to turn into a commercial venture".

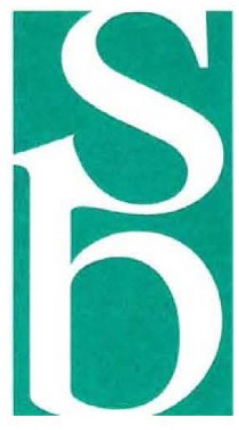

